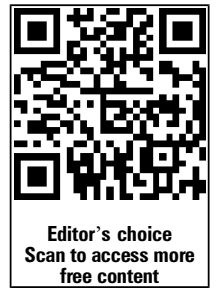

MRC Centre for Neuromuscular Diseases, UCL Institute of Neurology and the National Hospital for Neurology and Neurosurgery, London, UK

\section{Correspondence to} Professor Mary M Reilly, MRC Centre for Neuromuscular Diseases, UCL Institute of Neurology and the National Hospital for Neurology and Neurosurgery, Queen Square, London WC1N 3BG, UK; m.reilly@ucl.ac.uk

Accepted 10 March 2015 Published Online First

21 April 2015

\title{
A practical approach to the genetic neuropathies
}

\author{
Alexander M Rossor, Matthew R B Evans, Mary M Reilly
}

\begin{abstract}
Charcot-Marie-Tooth disease is the commonest inherited neuromuscular disease. It is characterised by degeneration of peripheral sensory and motor nerves and can be classified into axonal and demyelinating forms. This review provides a diagnostic approach to patients with suspected inherited neuropathy and an algorithm for genetic testing that includes recent advances in genetics such as next-generation sequencing. We also discuss important aspects of the longterm management of patients with inherited neuropathy.
\end{abstract}

\section{INTRODUCTION}

Genetic neuropathies encompass a range of diseases from those in which the neuropathy is the sole or predominant feature of the disease to those in which the neuropathy occurs as part of a multisystem disease, for example, Friedreich's ataxia (table 1). Next-generation sequencing has allowed us to identify a third group where genes that normally cause a different neurological syndrome or a complex syndrome, for example, hereditary spastic paraparesis secondary to REEP1 or atlastin 1 mutations, can rarely cause an isolated neuropathy.

We focus on those genetic neuropathies in which the neuropathy is the sole or predominant feature of the disease. This encompasses a group of diseases, collectively referred to as Charcot-Marie-Tooth disease (CMT) and related disorders. ${ }^{1}$ The terms hereditary motor neuropathy and hereditary sensory (and autonomic) neuropathy refer to forms of CMT where the burden of the disease falls on either motor or sensory nerves and represent extremes of the CMT spectrum.

We provide a structured approach to diagnosing a genetic neuropathy using recent advances in genetic sequencing technology and we also outline several management issues that arise in the long-term management of patients with CMT and related disorders.

\section{DIAGNOSIS}

The first step in diagnosing CMT and related disorders is to establish whether the patient has a neuropathy. This is usually obvious clinically as most patients present with length-dependent weakness and sensory loss that begins in the feet and then slowly ascends to the level of the knees before the hands become involved. The clinical impression should be confirmed with nerve conduction studies. It is noteworthy that in some congenital motor neuropathies, the compound muscle action potentials may be normal due to collateral sprouting and, in this scenario, a neuropathy may only be diagnosed with electromyography.

\section{IS IT GENETIC?}

The diagnosis of a genetic neuropathy may be obvious in large families with multiple affected family members but can be challenging in adopted individuals and those from small families. A slowly progressive history over many years is the norm. The age of onset can usually be dated back to the first or second decade although one should be aware that for some axonal forms of CMT, such as those due to mutations in HSPB1, the age of onset can be as late as the fourth decade; ${ }^{2}$ in other forms of CMT2, especially the forms with as yet unidentified genes, the age of onset can be in the sixth or seventh decade. A developmental history is essential, specifically asking about the presence of contractures at birth, developmental milestones, ill-fitting shoes and poor performance in sport at school.

Nerve conduction studies are particularly helpful for diagnosing demyelinating CMT and typically show homogenous slowing in the commonest subtype, 
Table 1 Classification of the inherited neuropathies

1. The neuropathy is the sole or primary component of the disease

- CMT, HNPP, HSN, HMN, hereditary neuralgic amyotrophy

2. The neuropathy is part of a complex, multisystem disorder

Inherited ataxias

- Autosomal dominant: spinocerebellar ataxia

- Autosomal recessive: Friedreich's ataxia, vitamin E deficiency, ARSACS, AOA1

Inherited spastic paraplegia

- BSCL2, REEP1, Atlastin, KIF1A

Porphyrias

- AIP, variegate porphyria, hereditary coproporphyria

Disorders of lipid metabolism

- Lipoprotein deficiencies: Tangier's disease, abetalipoproteinaemia, cerebrotendinous xanthomatosis

- Leukodystrophies (metachromatic, Krabbe's disease, adrenoleukodystrophy)

- Peroxisomal disorders (Refsum's disease, Fabry's disease)

- Sphingomyelin lipidoses and gangliosidoses

Mitochondrial disorders

- MNGIE, NARP, SANDO

Defective DNA repair/maintenance

- Xeroderma pigmentosum, Cockayne's syndrome, ataxia telangiectasia

Other

- Neuroacanthocytosis, neurofibromatosis type 1 and 2, myotonic dystrophy, familial amyloid polyneuropathy

3. Multisystem disorders presenting as neuropathy and occasionally with neuropathy being the only sole manifestation

- Distal HMN due to REEP1 mutations

- HSN1 secondary to atlastin 1 mutations.

- Neuropathy secondary to MT-ATP 6 mutations.

AIP, acute intermittent porphyria; AOA1, ataxia with oculomotor apraxia type 1; ARSACS, autosomal recessive spinocerebellar ataxia of Charlevoix Saguenay; CMT, Charcot-Marie-Tooth disease; HMN, hereditary motor neuropathy; HNPP, hereditary neuropathy with liability to pressure palsies; HSN, hereditary sensory neuropathy; MNGIE, mitochondrial neurogastrointestinal encephalopathy syndrome; NARP, neuropathy, ataxia and retinitis pigmentosa; REEP1, receptor accessory protein 1; SANDO, sensory ataxia neuropathy dysarthria and ophthalmoplegia.

CMT1A, where the median or ulnar motor conduction velocity is always $<38 \mathrm{~m} / \mathrm{s}^{3}$ Patchy slowing and conduction block more suggestive an acquired inflammatory neuropathy but can be seen in rare forms of CMT (see below) so a careful history is critical.

\section{CHAMELEONS}

An acute or subacute onset, patchy neuropathy with conduction block strongly suggests an acquired demyelinating neuropathy, such as chronic inflammatory demyelinating polyradiculoneuropathy. Over the last 5 years, however, it has been recognised that for a handful of genetic types of CMT (GJB1, MPZ, SH3TC2, SPTLC1 and FIG4) the neurophysiology may suggest an acquired inflammatory neuropathy. ${ }^{4-8}$ This is most commonly encountered for CMTX1 due to mutations in GJB1 and should be considered in patients with treatment-resistant chronic inflammatory demyelinating polyradiculoneuropathy. ${ }^{6}$

Other clinical markers that can help to differentiate genetic from acquired neuropathies include asymmetric weakness, cerebrospinal fluid (CSF) fluid examination and MRI of nerve roots. While these remain useful diagnostic tools, they are not absolute. For example, up to $7 \%$ of patients with CMT1A have a minor degree of asymmetry. ${ }^{9}$ Similarly, a CSF protein of up to $1 \mathrm{~g} / \mathrm{L}$ (but not $>2 \mathrm{~g} / \mathrm{L}$ ) and the presence of thickened nerve roots may occur in genetic demyelinating neuropathies such as CMT1A. ${ }^{10} 11$

If one takes a detailed history, almost all cases of CMT have a chronic and progressive course. The one exception is CMT due to homozygous or compound heterozygous mutations in FIG4 in which patients may develop acute weakness and wasting of one limb resembling motor neurone disease (often on the background of a chronic demyelinating neuropathy). ${ }^{4} 12$

\section{MIMICS}

Distal myopathies are a rare group of diseases that may be difficult to differentiate clinically from the distal hereditary motor neuropathies. Electromyography is the most useful investigation to distinguish the two conditions, although there may be some clinical clues in the upper limbs such as the early involvement of the intrinsic hand muscles in distal hereditary motor neuropathy (dHMN) as opposed to the forearm flexors in distal myopathy. ${ }^{13}$ It is important to differentiate the distal myopathies from $\mathrm{dHMN}$ as in the former cardiac screening is indicated to identify a cardiomyopathy. ${ }^{14}$

Foot deformities such as pes cavus are common in many genetic neuropathies; however, high arches are not always pathological. High foot arches and hammer toes resembling pes cavus may also occur in length-dependent muscle wasting from an acquired and potentially treatable neuropathy.

\section{DEFINING GENETIC NEUROPATHIES ACCORDING TO PHENOTYPE}

Having made a diagnosis of CMT and related disorders the next step is to classify the clinical phenotype as this helps to direct genetic testing and to interpret next generation sequencing data. Defining the clinical phenotype involves asking three main questions:

1. What is the likely mode of inheritance?

2. Is the neuropathy demyelinating or axonal?

3 . Is the neuropathy predominantly sensory, motor or mixed? 
Determining the mode of inheritance may be challenging in small families and sporadic cases. When the mode of inheritance is not obvious, as a general rule, autosomal dominant or de novo dominant inheritance is more common in Northern Europe and America, whereas autosomal recessive inheritance is more common in countries where consanguineous marriage is more prevalent. Male-to-male transmission excludes $\mathrm{X}$-linked inheritance. Strict maternal inheritance may indicate a mitochondrial DNA mutation, which may rarely cause CMT2. ${ }^{15}$

Having established the likely mode of inheritance, the next step is to determine whether the neuropathy is demyelinating or axonal using nerve conduction studies. Those with a conduction velocity of the median/ulnar nerve of $<38 \mathrm{~m} / \mathrm{s}$ are classified as demyelinating and those $>38 \mathrm{~m} / \mathrm{s}$ as axonal. ${ }^{1}$ This cut-off is particularly useful for identifying patients with the commonest form of CMT1, CMT1A, in whom all individuals have conduction velocities $<38 \mathrm{~m} / \mathrm{s}$. There is also a third category, intermediate CMT, where the conduction velocities are between 25 and $45 \mathrm{~m} / \mathrm{s}^{1}{ }^{1}$ (See box 1 ).

The third step in defining the phenotype is to determine the degree of motor and sensory involvement. Nerve conduction studies are essential as it is common for a patient with CMT to have significant sensory involvement on neurophysiology but minimal symptoms and clinical signs on examination. This is particularly relevant to axonal forms of CMT as it allows affected individuals to be classified as those that are motor predominant (hereditary motor neuropathy (HMN)), those that are sensory predominant (hereditary sensory neuropathy (HSN)) and those with mixed motor and sensory involvement (CMT2). In reality, this is a spectrum and many of the HMN genes such as HSPB1 cause both HMN and CMT2; it is rare to find a patient with HSN who does not eventually develop weakness. ${ }^{2} 5$

Nerve biopsy now has almost no role in the evaluation of a patient with CMT and should be reserved for complex patients referred to tertiary neuromuscular clinics especially where there is clinical suspicion of an acquired and potentially treatable inflammatory neuropathy.

Having established the clinical phenotype, the next step is to determine the causative gene. Although this may seem daunting for a disease where there are over 80 known genes, it is worth remembering that most patients with CMT (> 60\% in the UK) have one of five gene mutations: the duplication of the $17 \mathrm{p}$ chromosome or mutations of PMP22, GJB1, MPZ and $M F N 2^{17} 18$ (see figure 1). Of these, the $17 \mathrm{p}$ duplication is the commonest, accounting for at least $90 \%$ of CMT1. ${ }^{17} 18$ The commonest autosomal recessive form of CMT seen in the UK is CMT4C due to mutations in SH3TC2. Table 2 provides a comprehensive summary of all known genes in CMT and related disorders at the time of writing.

\section{Box 1 CMT categorisation}

Charcot-Marie-Tooth disease (CMT) 1 refers to autosomal dominant demyelinating CMT and CMT4 refers to autosomal recessive demyelinating CMT. Autosomal dominant axonal CMT is referred to as CMT2 and if recessive as autosomal recessive-CMT2. If the neuropathy is axonal and motor predominant, it is termed hereditary motor neuropathy and if it is predominantly sensory it is termed hereditary sensory neuropathy. X-linked CMT is referred to as CMTX. Intermediate CMT is referred to as dominant intermediate-CMT if dominant and recessive intermediate-CMT if recessive. Dejerine-Sottas (or CMT3) and congenital hypomyelinating neuropathy are historic terms that describe congenital or early onset, severe inherited neuropathies. These are often due to de novo dominant mutations in peripheral myelin protein 22, myelin protein zero and EGR2 and to avoid confusion it is probably easier to refer to them as severe early-onset demyelinating neuropathies (usually CMT1 or in rare cases of autosomal recessive inheritance, CMT4). ${ }^{16}$ Roussy-Levy syndrome is now an outdated term that describes the association of CMT and tremor.

\section{CMT1A (17p duplication)}

CMT1A is the commonest genetic subtype of $\mathrm{CMT}^{17} 18$ and is a fully penetrant disease on electrophysiological examination. ${ }^{3}$ The clinical presentation is of distal limb weakness most evident in the lower limbs with reduced or absent deep tendon reflexes. Sensory symptoms are often minimal although length-dependent sensory loss on examination is a common feature and may affect all sensory modalities. ${ }^{19}$ Common reasons for presentation include delayed walking, inability to run despite previously normal developmental milestones and the presence of foot deformities. ${ }^{19}$

CMT1A is a slowly progressive disease and usually has no effect on life span. ${ }^{20}$ Most patients with CMT1A remain fully ambulant but may require orthotic support with increasing disease duration.

The vast majority of cases of CMT1A are due to a duplication of approximately 1.5 million base pairs on chromosome $17 \mathrm{p} 11.1-\mathrm{p} 12^{21}$ containing the peripheral myelin protein 22 (PMP22) gene.

Point mutations in PMP22 are a much less common cause of CMT than the $17 \mathrm{p}$ duplication $(1 \%-5 \%)$ and may cause CMT1A, hereditary neuropathy with liability to pressure palsies (HNPP) and a more severe form of CMT1 especially in de novo dominant cases. ${ }^{22}$

\section{HNPP (17p deletion)}

Deletions of the same 1.5 million base pairs on chromosome $17 \mathrm{p}$ duplicated in CMT1A result in $\mathrm{HNPP}^{23}$ Patients with HNPP present with recurrent, severe pressure palsies often occurring after minimal injury. The disease is often obvious on nerve conduction studies, 


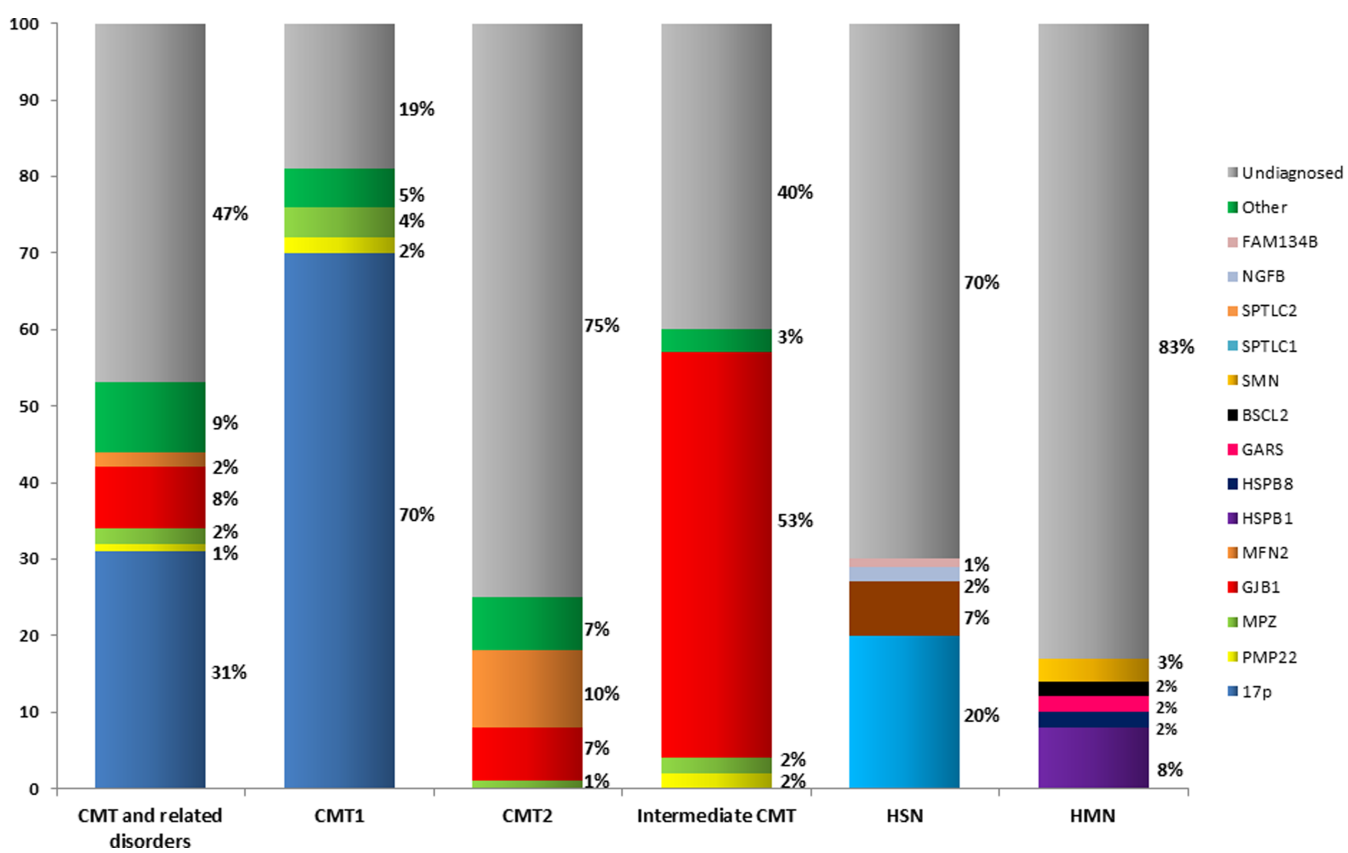

Figure 1 Genetic diagnoses in Charcot-Marie-Tooth disease (CMT) and related disorders in patients attending a specialist CMT clinic in the UK (inherited neuropathy clinic, National Hospital for Neurology and Neurosurgery, Queen Square). ${ }^{17}$ It can be seen that for the majority of patients with CMT2, hereditary sensory neuropathy (HSN) and hereditary motor neuropathy (HMN) the underlying genetic defect is unknown. BSCL, Berardinelli-Seip congenital lipodystrophy; GARS, glycyl tRNA synthetase; HSPB8, heat shock protein 22kDa protein 8; MFN, mitofusin 2; MPZ, myelin protein zero; NGFB, nerve growth factor $\beta$; PMP, peripheral myelin protein; SPTLC, serine palmitoyltransferase, long-chain; SMN, survival of motor neuron.

which show a background neuropathy characterised by reduced sensory nerve action potentials, borderline motor and sensory nerve conduction velocities and conduction block at sites of compression. ${ }^{24}$ As such, testing for the $17 \mathrm{p}$ deletion is not recommended in isolated compressive mononeuropathies.

The mainstay of management in HNPP involves avoiding situations which may predispose to nerve compression. This includes avoiding excessive alcohol and ensuring meticulous intraoperative positioning during surgery. It is currently unclear when or whether to offer patients with a rapidly progressive and symptomatic carpal tunnel syndrome and HNPP surgical decompression. There have been case reports of clinical improvement after surgery and it is our usual practice to avoid surgery unless there is evidence of progressive motor weakness or very severe, typical and prolonged sensory symptoms. ${ }^{25}$

\section{CMT1B (myelin protein zero (MPZ))}

Mutations in MPZ commonly result in an autosomal dominant neuropathy and are estimated to account for $5 \%$ of cases of $\mathrm{CMT}^{26} \mathrm{MPZ}$ mutations show considerable phenotypical heterogeneity ranging from a severe congenital demyelinating neuropathy with significant symptomatic large fibre sensory involvement through to a late-onset mild neuropathy. This heterogeneity is also reflected in the neurophysiology which may show very slow nerve conduction velocity of $<10 \mathrm{~m} / \mathrm{s}$ through to normal conduction velocities.

\section{CMT2A (mitofusin 2 (MFN2))}

Mutations in MFN2 are the commonest known cause of autosomal dominant CMT2. ${ }^{27}$ The age of onset is usually in the first decade with a classical phenotype of foot deformity and distal weakness. The disease course is usually aggressive in comparison with other forms of CMT2 and CMT1 with many patients requiring a wheelchair by the second decade. Additional clinical features include the variable presence of optic neuropathy and brisk reflexes. ${ }^{28}$ Many MFN2 mutations are novel, many are de novo and there are many polymorphisms all of which contribute to diagnostic difficulties.

\section{CMTX1 (connexin 32, GJB1)}

CMTX1 is the second most common cause of CMT after CMT1A. ${ }^{29}$ The disease can be considered to be $\mathrm{X}$-linked dominant with both men and women affected but with men affected more severely.

Disease onset in men is in the first or second decade with distal weakness and loss of sensation. Positive sensory symptoms including pain are a common feature $^{29}$ and patients may display the 'split hand' phenomenon characterised by increased wasting and weakness of the abductor pollicis brevis compared with the first dorsal interosseous muscle.

The nerve conduction velocities in CMTX1 usually show a patchy demyelinating neuropathy although in women the conduction velocities may be in the axonal range. ${ }^{29}$ 
Table 2 All known disease genes in CMT and related disorders

\begin{tabular}{|c|c|c|}
\hline Type (OMIM number) & Gene & Phenotype \\
\hline \multicolumn{3}{|l|}{ Autosomal dominant CMT1 } \\
\hline CMT1A (118220) & $\begin{array}{l}\text { 17p dup. (PMP22) } \\
\text { PMP22 point mutation }\end{array}$ & $\begin{array}{l}\text { Classic CMT1 } \\
\text { Classic CMT1, DSD, CHN (rarely recessive) }\end{array}$ \\
\hline CMT1B (118200) & $M P Z$ & CMT1, DSD, CHN, CMT2 (rarely recessive) \\
\hline CMT1C (601098) & LITAF & Classic CMT1 \\
\hline CMT1D (607678) & $E G R 2$ & Classic CMT1, DSD, CHN \\
\hline CMT1F (607734) & NEFL & $\begin{array}{l}\text { CMT2 but can have slow MCV in the CMT1 range (rarely } \\
\text { recessive) }\end{array}$ \\
\hline CMT1 plus (614434) & FBLN5 & Macular degeneration, cutis laxa, HMN, slow NCV \\
\hline SNCV/CMT1 (608236) & ARHGEF10 & Asymptomatic slow conduction velocities \\
\hline \multicolumn{3}{|c|}{ Hereditary neuropathy with liability to pressure palsies } \\
\hline HNPP (162500) & $\begin{array}{l}\text { 17p del. (PMP22) } \\
\text { PMP22 point mutation }\end{array}$ & $\begin{array}{l}\text { Typical HNPP } \\
\text { Typical HNPP }\end{array}$ \\
\hline \multicolumn{3}{|l|}{ Autosomal recessive CMT1 } \\
\hline CMT4A (214400) & GDAP1 & $\begin{array}{l}\text { CMT2, usually severe early onset } \\
\text { Vocal cord and diaphragmatic paralysis described }\end{array}$ \\
\hline CMT4B1 (601382) & MTMR2 & Severe CMT1, facial, bulbar, focally folded myelin \\
\hline CMT4B2 (604563) & SBF2 & Severe CMT1, glaucoma, focally folded myelin \\
\hline CMT4B3 (615284) & SBF1 & CMT1, focally folded myelin \\
\hline CMT4C (601596) & SH3TC2 & Severe CMT1, scoliosis, cytoplasmic inclusions \\
\hline CMT4D or HMSNL (601455) & NDRG1 & Severe CMT1, gypsy, deafness, tongue atrophy \\
\hline CMT4E (605253) & $E G R 2$ & CMT1, DSD, CHN phenotype \\
\hline CMT4F (614895) & $P R X$ & CMT1, predominantly sensory, focally folded myelin \\
\hline CMT4G or HMSN Russe (605285) & $H K 1$ & Severe early-onset CMT1, gypsy \\
\hline CMT4H (609311) & FGD4 (Frabin) & Classic CMT1 \\
\hline CMT4J (611228) & FIG4 & CMT1, predominantly motor, progressive \\
\hline CCFDN (604168) & CTDP1 & CMT1, gypsy, cataracts, dysmorphic features \\
\hline CMT4 & SURF-1 & $\begin{array}{l}\text { CMT1, encephalopathy, ataxia, reduced life span, Leigh's } \\
\text { syndrome }\end{array}$ \\
\hline \multicolumn{3}{|l|}{ Autosomal dominant CMT2 } \\
\hline CMT2A (609260) & MFN2 & CMT2, progressive, optic atrophy (rarely recessive) \\
\hline CMT2B or HSAN1B (600882) & $R A B 7$ & CMT2 with sensory complications (ulcero mutilating) \\
\hline CMT2C (606071) & TRPV4 & CMT2, vocal cord paralysis \\
\hline CMT2D (601472) & GARS & CMT2 with predominant hand wasting \\
\hline CMT2E (607684) & NEFL & $\begin{array}{l}\text { CMT2 but can have nerve conduction velocities in the CMT1 } \\
\text { range (rarely recessive) }\end{array}$ \\
\hline CMT2F (606595) & HSPB 1 & Motor-predominant CMT2 \\
\hline CMT2I (607677) & $M P Z$ & Late-onset CMT2 \\
\hline CMT2J (607736) & $M P Z$ & CMT2 with hearing loss and pupillary abnormalities \\
\hline CMT2K (607831) & GDAP1 & Late-onset CMT2 (dominant), severe CMT2 (recessive) \\
\hline CMT2L (608673) & HSPB8 & Motor-predominant CMT2 \\
\hline CMTDIB or CMT2M (606482) & DNM2 & Intermediate CMT or CMT2, cataracts, ophthalmoplegia, ptosis \\
\hline CMT2N (613287) & AARS & Classic CMT2 \\
\hline CMT2P (614436) & LRSAM1 & Mild sensory-predominant CMT2 (dominant and recessive) \\
\hline CMT2Q (615025) & DHTKD1 & CMT2 \\
\hline HMSNP (604484) & TFG & CMT2 with proximal involvement \\
\hline CMT2 & MARS & Late-onset CMT2 \\
\hline CMT2 & HARS & CMT2 \\
\hline CMT2 & $V C P$ & CMT2 \\
\hline SPG10 (604187) & KIF5A & CMT, hereditary spastic paraplegia \\
\hline CMT2 & MT-ATP6 & CMT2, pyramidal signs, relapsing \\
\hline \multicolumn{3}{|l|}{ Autosomal recessive CMT2 } \\
\hline CMT2B1 (605588) & LMNA & CMT2 rapid progression \\
\hline
\end{tabular}




\section{REVIEW}

Table 2 Continued

\begin{tabular}{|c|c|c|}
\hline Type (OMIM number) & Gene & Phenotype \\
\hline CMT2B2 (605589) & MED25 & Classic CMT2 \\
\hline NMAN (137200) & HINT1 & Neuromyotonia and axonal neuropathy, motor predominant \\
\hline CMT2R (615490) & TRIM2 & Infantile-onset CMT2 \\
\hline AR-CMT2 & IGHMBP2 & CMT2 \\
\hline AR-CMT2 & $H S J 1$ & CMT2 \\
\hline \multicolumn{3}{|l|}{ X-linked CMT } \\
\hline CMTX1 (302800) & GJB1 & Males CMT1 (patchy NCV); females CMT2 \\
\hline CMTX4 or Cowchock'o syndrome (310490) & AlFM1 & $\begin{array}{l}\text { CMT2, infantile onset, developmental delay, deafness, learning } \\
\text { difficulties }\end{array}$ \\
\hline CMTX5 (311070) & PRPS1 & CMT2, deafness, optic atrophy \\
\hline CMTX6 (300905) & PDK3 & CMT2 \\
\hline \multicolumn{3}{|l|}{ Dominant intermediate CMT } \\
\hline CMTDIB or CMT2M (606482) & DNM2 & Intermediate CMT or CMT2, cataracts, ophthalmoplegia, ptosis \\
\hline CMTDIC (608323) & YARS & Intermediate CMT \\
\hline CMTDID (607791) & $M P Z$ & Intermediate CMT \\
\hline CMTDIE (614455) & IFN2 & $\begin{array}{l}\text { Intermediate CMT, focal segmental glomerulosclerosis, } \\
\text { end-stage renal failure }\end{array}$ \\
\hline CMTD1F (615185) & GNB4 & Intermediate CMT \\
\hline \multicolumn{3}{|l|}{ Recessive intermediate CMT } \\
\hline CMTRIA (608340) & GDAP1 & Intermediate CMT \\
\hline CMTRIB (613641) & KARS & Intermediate CMT, learning difficulty, vestibular schwannoma \\
\hline CMTRIC (615376) & PLEKHG5 & Intermediate CMT, SMA \\
\hline CMTRID (616039) & COX6A1 & Intermediate CMT, onset first decade \\
\hline \multicolumn{3}{|l|}{ Hereditary motor neuropathy } \\
\hline HMN2A (158590) & HSPB8 & Classical HMN, dominant \\
\hline HMN2B (608634) & HSPB 1 & Classical HMN, dominant \\
\hline HMN2C (613376) & HSPB3 & Classical HMN, dominant \\
\hline HMN2D (615575) & FBX038 & Classical HMN, dominant \\
\hline HMN with pyramidal features or ALS4 (602433) & SETX & HMN with pyramidal signs, dominant \\
\hline DSMA5 (614881) & DNAJB2 (HSJ1) & Classical HMN, recessive \\
\hline HMN5A (600794) or SPG17 (270685) & BSCL2 & $\begin{array}{l}\text { Predominant hand wasting, Silver syndrome but can have } \\
\text { sensory involvement as in CMT2D, dominant }\end{array}$ \\
\hline HMN5A (600794) & GARS & Predominant hand wasting, dominant \\
\hline HMN5B (614751) or SPG31 (610250) & REEP1 & Predominant hand wasting, pyramidal signs, dominant \\
\hline HMN6 or SMARD1 (604320) & IGHMBP2 & Infantile onset, respiratory distress, recessive \\
\hline SMARD2 or SMAX & LAS1L & Infantile onset, respiratory distress, $\mathrm{X}$-linked recessive \\
\hline HMN7A (158580) & SLC5A7 & Classical HMN, vocal cord palsy, dominant \\
\hline HMN7B (607641) & DCTN1 & HMN, bulbar and facial weakness, dominant \\
\hline SMAX3 (300489) & ATP7A & Classical HMN, X-linked \\
\hline SMALED (158600) & DYNC1H1 & $\begin{array}{l}\text { Congenital, contractures, lower-limb predominant, pyramidal } \\
\text { signs, cortical migration defects, learning difficulties, dominant }\end{array}$ \\
\hline SMALED2 (615290) & $B / C D 2$ & $\begin{array}{l}\text { Congenital, contractures, lower-limb predominant, pyramidal } \\
\text { signs, dominant }\end{array}$ \\
\hline PNMHH (614369) & MYH14 & $\begin{array}{l}\text { Typical HMN, distal myopathy, hoarseness, hearing loss, } \\
\text { dominant }\end{array}$ \\
\hline SPSMA (181405) & TRPV4 & HMN, scapular winging, vocal cord palsy, dominant \\
\hline HMN & AARS & Typical HMN, dominant \\
\hline HMN & HINT1 & HMN with neuromyotonia, recessive \\
\hline \multicolumn{3}{|c|}{ Hereditary sensory neuropathy (also called Hereditary sensory and autonomic neuropathy (HSAN)) } \\
\hline HSAN1A (162400) & SPTLC1 & HSN with sensory complications (ulcero mutilating), dominant \\
\hline HSAN1C (613640) & SPTLC2 & HSN with sensory complications (ulcero mutilating), dominant \\
\hline CMT2B (600882) & RAB7 & HSN with sensory complications (ulcero mutilating), dominant \\
\hline
\end{tabular}


Table 2 Continued

\begin{tabular}{|c|c|c|}
\hline Type (OMIM number) & Gene & Phenotype \\
\hline HSN1D (613708) or SPG3A (182600) & ATL1 & $\begin{array}{l}\text { HSN with sensory complications (ulcero mutilating), spasticity, } \\
\text { dominant }\end{array}$ \\
\hline HSN1E (614116) & DNMT1 & HSN, hearing loss, dementia, dominant \\
\hline HSN1F (615632) & ATL3 & HSN, bone destruction, dominant \\
\hline HSAN2A (201300) & WNK1 & HSN with sensory complications (ulcero mutilating), recessive \\
\hline HSAN2B or HSAN1B (613115) & FAM134B & HSN with sensory complications (ulcero mutilating), recessive \\
\hline HSN2C (614213) or SPG30 (610357) & KIF1A & HSN with sensory complications (ulcero mutilating), recessive \\
\hline HSAN3, familial dysautonomia or Rileymia or Rileyt & IKBKAP & $\begin{array}{l}\text { Ashkenazi Jewish, autonomic dysfunction, HSN, absent } \\
\text { fungiform papillae, recessive }\end{array}$ \\
\hline $\begin{array}{l}\text { Insensitivity to pain (24300), paroxysmal extreme pain } \\
\text { disorder (167400), primary erythermalgia (133020), } \\
\text { small-fibre neuropathy }\end{array}$ & SCN9A & $\begin{array}{l}\text { Recessive: insensitivity to pain } \\
\text { Dominant: paroxysmal extreme pain disorder, primary } \\
\text { erythermalgia, small fibre neuropathy }\end{array}$ \\
\hline CIPA or HSAN4 (256800) & NTRK1 & Congenital insensitivity to pain with anhydrosis, recessive \\
\hline HSAN5 (608654) & $N G F-B$ & Insensitivity to pain, recessive \\
\hline HSAN6 (614653) & DST & $\begin{array}{l}\text { Ashkenazi Jewish, autonomic dysfunction, HSN, absent } \\
\text { fungiform papillae, death by age } 2 \text {, recessive }\end{array}$ \\
\hline HSAN7 (615548) & SCN11A & $\begin{array}{l}\text { Congenital insensitivity to pain with hyperhidrosis and } \\
\text { gastrointestinal dysfunction, dominant }\end{array}$ \\
\hline HSAN and dementia & PRNP & Autonomic dysfunction, sensory loss, dementia, dominant \\
\hline $\begin{array}{l}\text { Hereditary sensory neuropathy with spastic paraplegia } \\
(256840)\end{array}$ & CCT5 & $\begin{array}{l}\text { HSN with sensory complications (ulcero mutilating) and spastic } \\
\text { paraplegia, recessive }\end{array}$ \\
\hline
\end{tabular}

CHN, congenital hypomyelinating neuropathy; CMT, Charcot-Marie-Tooth disease; DSD, Dejerine-Sottas disease; HMN, hereditary motor neuropathy; HNPP, hereditary neuropathy with liability to pressure palsies; HSN, hereditary sensory neuropathy; MCV, motor conduction velocity; NCV, nerve conduction velocity; SMA, spinal muscular atrophy; SNCV, slowed nerve conduction velocity.

Connexin 32 is also expressed in the central nervous system and may cause white matter lesions on brain MRI that are usually but not always asymptomatic. ${ }^{29}$

\section{CMT4C (SH3TC2)}

Homozygous or compound heterozygous mutations in SH3TC2 are the commonest cause of autosomal recessive demyelinating CMT (CMT4) in the UK and should be considered in sporadic cases of demyelinating CMT. Affected individuals present in the first decade of life and scoliosis is a common feature and may be severe enough to warrant surgery. ${ }^{7}$ Median nerve conduction velocities range from 4 to $37 \mathrm{~m} / \mathrm{s}^{30}$

\section{GENETIC TESTING IN CMT, THE OLD AND THE NEW}

Genetic testing for CMT and many other neurological conditions has traditionally involved sequential testing of individual genes using Sanger sequencing. In this scenario, the most promising candidate gene after careful phenotyping is analysed and if negative the next most likely candidate is tested. This method is time and cost effective for CMT1A due to the $17 \mathrm{p}$ duplication but can be very frustrating, expensive and ultimately futile in axonal CMT, where each causative gene is individually rare and in as many as $75 \%$ of cases the disease gene is unknown (see figure 1$){ }^{17}$

The advent of multiple parallel or next generation sequencing has transformed the approach to genetic testing in $\mathrm{CMT}^{31}$ The technology allows the mass sequencing of a selection of genes (panels), the exome (containing only the protein encoding sequences) or the whole genome in a matter of days. There is a compromise, however, whereby for a given cost, either a small number of genes can be screened with good coverage (depth) or a large number of genes, for example, a whole exome can be screened but with less read depth so that the chance of missing a pathogenic mutation increases. It is our current practice to screen for mutations in patients with CMT and related disorders using targeted panels except in the case of CMT1 where we advocate testing for the $17 \mathrm{p}$ duplication first as it is cheap and the hit rate is high (see figure 2). This is a rapidly advancing field, however, and it is likely that as the technology advances and the cost falls, whole exome (and eventually whole genome) sequencing will replace disease-specific panels.

We are aware that some clinical geneticists, faced with a patient with clinically evident CMT, proceed directly to testing for the $17 \mathrm{p}$ duplication (as it is the commonest cause) and only undertake neurophysiology if this is negative. We do not advocate this approach for two main reasons (except perhaps in children where neurophysiology may be poorly tolerated and the genetic diagnosis of CMT1A has been confirmed in another family member). First, even though CMT1A is the commonest cause of CMT, it still only accounts for $<40 \%$ of all cases of inherited neuropathy and so testing for the $17 \mathrm{p}$ duplication without differentiating demyelinating from axonal types equates to a significant unnecessary expenditure. Second, a diagnosis of a particular type of CMT 


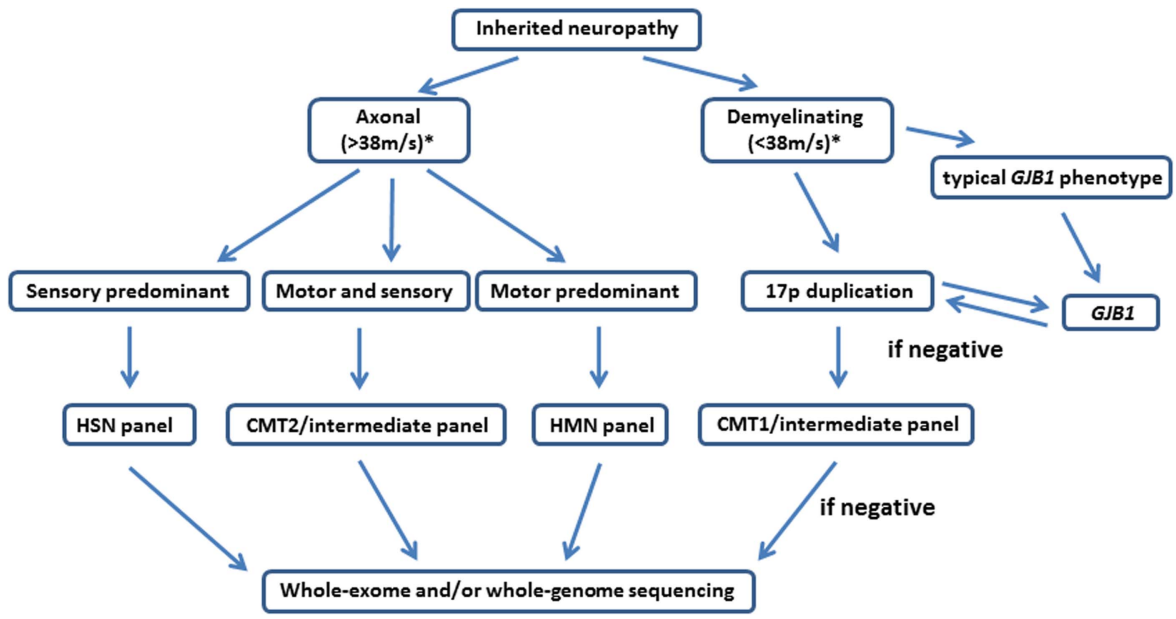

Figure 2 Suggested algorithm for genetic testing in Charcot-Marie-Tooth disease (CMT) and related disorders in the age of disease-specific gene panels. *Motor nerve conduction velocity. HMN, hereditary motor neuropathy; HSN, hereditary sensory neuropathy.

depends on validating the genetic results and one of the important tools in validation is whether the phenotype fits. As the clinical features of different genetic types of CMT are often very similar, neurophysiology is an essential part of the phenotyping (box 2).

\section{CHALLENGES OF NEXT-GENERATION SEQUENCING FOR THE PRACTISING NEUROLOGIST: DETERMINING THE PATHOGENICITY OF A NOVEL MUTATION}

One of the main challenges of next generation sequencing technology is in the bioinformatics analysis and the interpretation of the large number of genetic variants in known pathogenic genes. The average person has 400 potentially pathogenic variants in their exome. ${ }^{33}$ Thus, when a clinician requests a CMT panel encompassing up to 50 genes, it is usual to find several potentially pathogenic variants in more than one gene. Determining which one is the

Box 2 Practical limitations of next-generation sequencing

Next-generation sequencing technology currently cannot reliably detect large exonic duplications and deletions such as the 17p duplication (Charcot-Marie-Tooth disease (CMT) 1A) and deletion (hereditary neuropathy with liability to pressure palsies). Multiplex ligation-dependent probe amplification (MLPA) is the investigation of choice for detecting such genomic rearrangements. MPLA is also useful for the detection of exonic deletions in other CMT genes, for example, in the UK, there is a common founder deletion in mitofusin 2 that, in association with a point mutation on the other allele, is a cause of autosomal recessive CMT2. ${ }^{32}$ pathogenic variant is a new skill that is increasingly being asked of the clinical neurologist. It is our practice to approach this question by evaluating (1) the clinical phenotype, (2) segregation of the mutation with the disease and (3) the molecular properties and frequency of the mutation in healthy controls.

\section{Phenotype}

When first evaluating a potentially pathogenic mutation, it is important to determine whether the patient's phenotype fits with what has already been described for the gene. For example, a novel missense mutation in MFN2 (a gene with a large number of polymorphisms) is unlikely to be the cause of a demyelinating neuropathy. If a mutation has previously been published for the patient's phenotype then this often but not always provides further evidence for the pathogenicity of a mutation. The caveat to this is that many published genes and mutations have only been described in single families and doubt therefore remains as to their true pathogenicity. With next-generation sequencing, we are also seeing CMT caused by genes that traditionally cause a different phenotype, for example, REEP1 causing distal hereditary motor neuropathy rather than hereditary spastic paraparesis so the broadening phenotypes seen with different genes needs to be kept in mind.

\section{Segregation in families}

Perhaps the most useful test for determining the pathogenicity of a mutation is to determine whether the mutation segregates with the disease. This can be time consuming and requires both affected and unaffected family members to be examined often including performing neurophysiology and for further DNA to be collected and tested. As some forms of axonal neuropathy may manifest after the fourth decade, care must be taken in labelling a family member as unaffected. In most cases it is necessary to 
reserve judgement on unaffected individuals unless very elderly or in a family with a large number of affected individuals with a similar early age of onset.

\section{Molecular and epidemiological properties of the mutation}

Several predictive programmes, freely available online (eg, Sibyl, PON-P2, Predict SNP, META-SNP) aim to predict the pathogenicity of a missense mutation. While these programmes can help, it is worth remembering that for many known pathogenic mutations (eg, pathogenic HSPB1 mutations) some of these programmes have failed to predict pathogenicity.

An additional step in determining the pathogenicity of a mutation is to discover whether the substituted amino acid is conserved across species as mutations in amino acids that are not conserved are less likely to be pathogenic. This analysis can be easily performed using the freely available polyphen-2 software (http:// genetics.bwh.harvard.edu/pph2/). Finally, searching for novel mutations on public databases of single nucleotide polymorphisms (eg, the exome variant server) will reveal whether the novel variant is present in 'healthy controls'. The presence of a variant in one of these databases should not be taken as absolute evidence that the variant is non-pathogenic as it is likely that several pathogenic variants have been miscategorised as single nucleotide polymorphisms. The increasing information about polymorphisms in different ethnic groups being gained from next-generation sequencing will greatly help in the future to determine whether a mutation is pathogenic.

\section{MANAGEMENT}

While achieving a genetic diagnosis is an important part of the clinical evaluation of a patient with a genetic neuropathy there are several other clinical aspects to be addressed during the outpatient consultation.

\section{Foot care}

CMT and related disorders share many similarities to diabetic polyneuropathy. It is our practice to provide our patients with general advice on foot care and to refer all patients with significant sensory involvement to a chiropodist in order to prevent foot ulcers.

\section{Orthopaedic aspects of CMT}

There are three main reasons for orthopaedic intervention in CMT and related disorders. These include (1) scoliosis, (2) hip dysplasia and (3) foot and ankle surgery. ${ }^{34}$ Scoliosis occurs in $26 \%-37 \%$ of patients with CMT but rarely requires surgical intervention unless there is rapid progression or the degree of deformity extends beyond $45^{\circ}$.

The prevalence of hip dysplasia in CMT and related disorders is about $8 \%{ }^{35}$ and is more common in CMT1. X-rays of the hips and pelvis should therefore be requested in patients with a significant deterioration in their gait or if transitioning from paediatric to adult services without previous imaging.

Foot and ankle manifestations

There are three common foot deformities in CMT and related disorders: claw toes, forefoot (pes) cavus and hind foot varus (ankle inversion; see figure 3). For any patient with foot deformity or weakness we recommend referral to a physiotherapist and/or orthotist with an interest in CMT.

\section{Conservative management of foot deformity in CMT}

The non-operative management of foot deformity in CMT includes gastrocnemius stretching exercises and the provision of insoles or ankle-foot orthoses to reduce foot pain and to improve ambulation. ${ }^{36}$ There is a wide variety of ankle-foot orthoses offering different degrees of support and rigidity (figure 4).

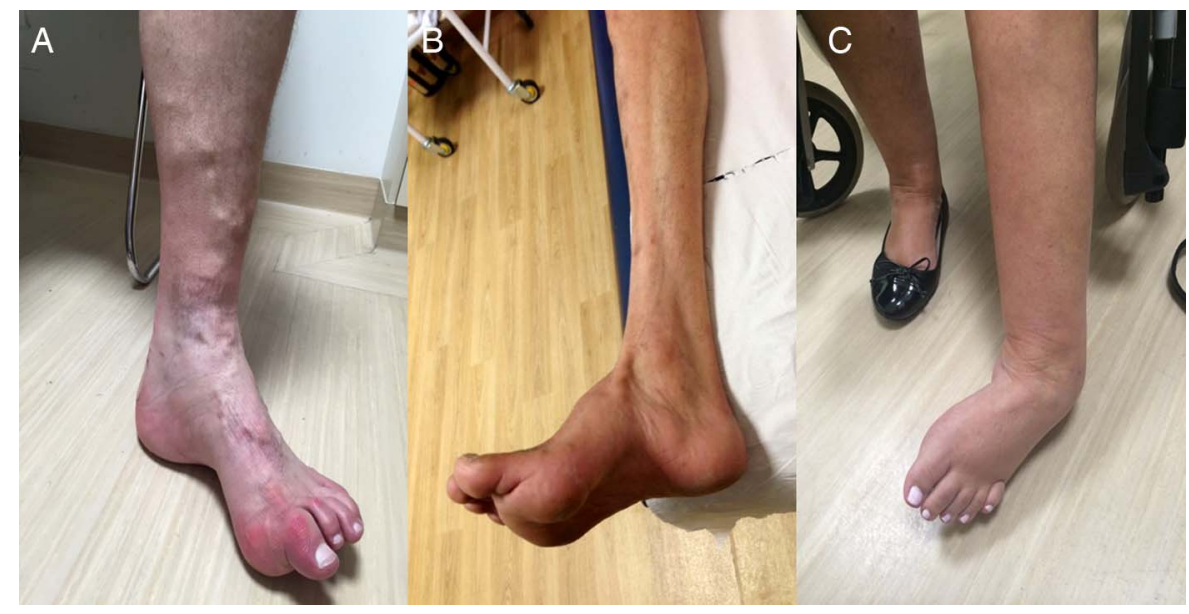

Figure 3 Common foot deformities in Charcot-Marie-Tooth disease and related disorders. (A) Claw toes, (B) pes cavus and (C) hind foot varus deformities of the feet. 


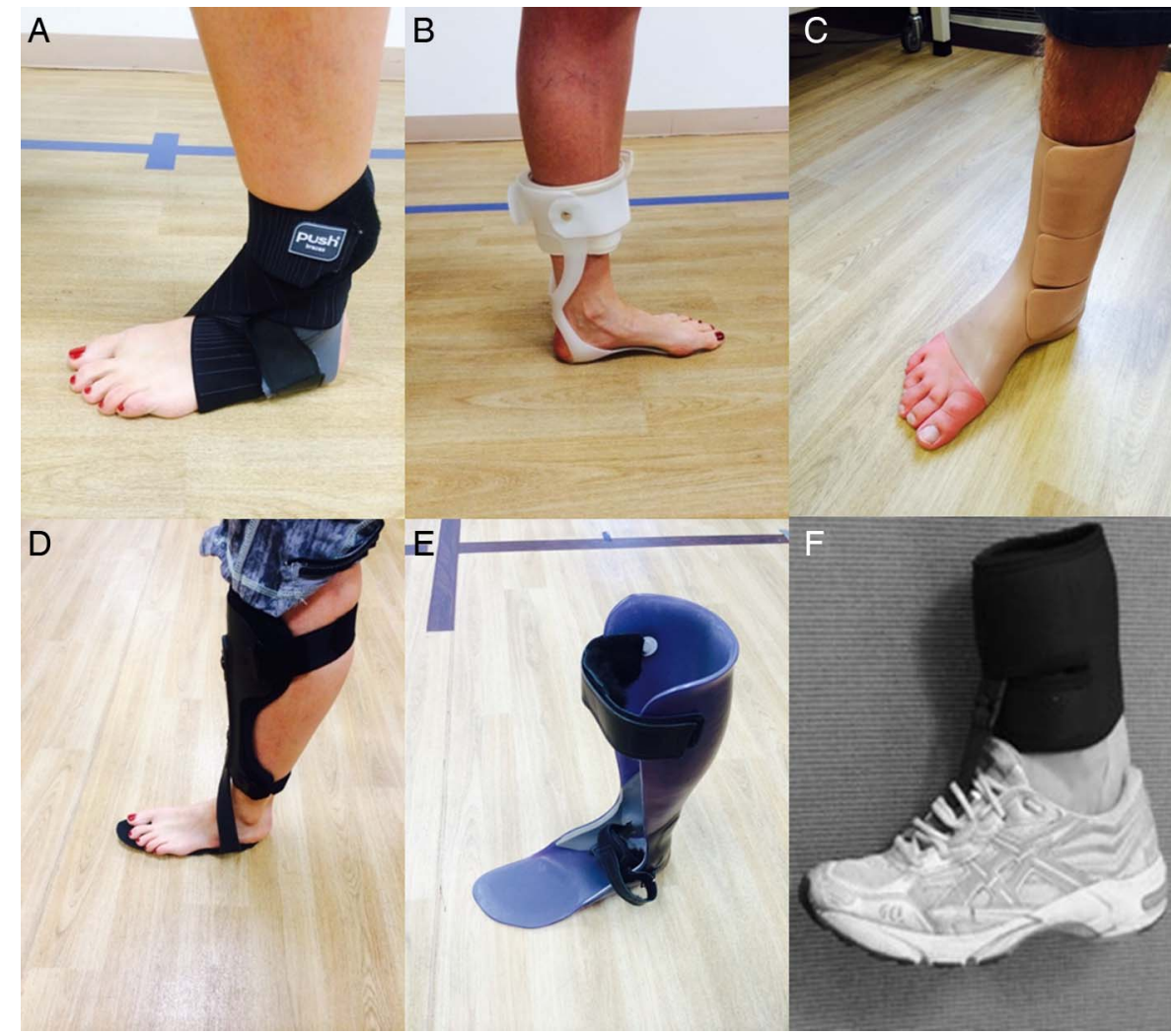

Figure 4 Various lower limb ankle orthoses used in the management of distal lower limb muscle weakness in patients with inherited neuropathies. (A) Push Aequi ankle brace; (B) injection-moulded polyethylene Swedish ankle-foot orthosis; (C) silicone ankle-foot orthosis; (D) matrix max carbon fibre ankle-foot orthosis; (E) rigid ankle-foot orthosis; (F) foot-up ankle-foot orthosis.

Surgical management of foot deformity in CMT

If conservative management of foot deformity is unsuccessful, it may be necessary to consider surgical intervention. There are three main types of foot operation for patients with CMT aimed at improving ambulation. They are soft tissue corrections, osteotomies and fusions (see figure 5). ${ }^{34}$

\section{Driving}

In any patient with peripheral neuropathy and significant weakness or sensory involvement, it is important to remind them of the need to inform the driving authorities of their condition. For those patients in whom driving is becoming increasingly problematic, they may be directed to the regional driving assessment centres (http://www.rdac.co.uk/) who offer a self-funded disability driving assessment.

\section{Genetic counselling}

There are several scenarios in CMT that require special consideration before offering genetic testing. First, presymptomatic testing of children aged $<18$ years at risk of developing CMT is not usually performed. Our current practice is to only offer testing of at risk children when they are thought to be affected while acknowledging that the early symptoms may be very subtle with a suspicion of minimal walking difficulties. In this early symptomatic scenario, parents sometimes opt for a follow-up appointment to monitor the child rather than further tests at this stage. In children who are clinically affected, we usually advocate undertaking neurophysiology first and proceed to appropriate genetic testing if the neurophysiology is abnormal. In a family where the genetic diagnosis is known to be CMT1A, especially in young children where neurophysiology may not be tolerated as well, it is appropriate to proceed directly to testing for the chromosome 17 duplication.

Second, a relatively common scenario in both specialist neuromuscular and general neurology clinics is for a patient with CMT to be referred from primary care as they wish to start a family. Some of these patients wish to undergo antenatal testing or preimplantation genetic diagnosis. Genetic counselling for prenatal diagnosis of CMT is becoming increasingly complex. In the case of the common pathogenic mutations such as the $17 \mathrm{p}$ duplication, identifying a carrier prenatally is straightforward. Unfortunately, many mutations identified in routine genetic testing are novel, and it can be difficult trying to decide if they are pathogenic or simply polymorphisms. In the UK, each individual causative gene must be approved by a panel before it can be offered for preimplantation genetic diagnosis.

\section{SUMMARY}

Most general neurologists will come across CMT and related disorders in their daily practice. A positive 


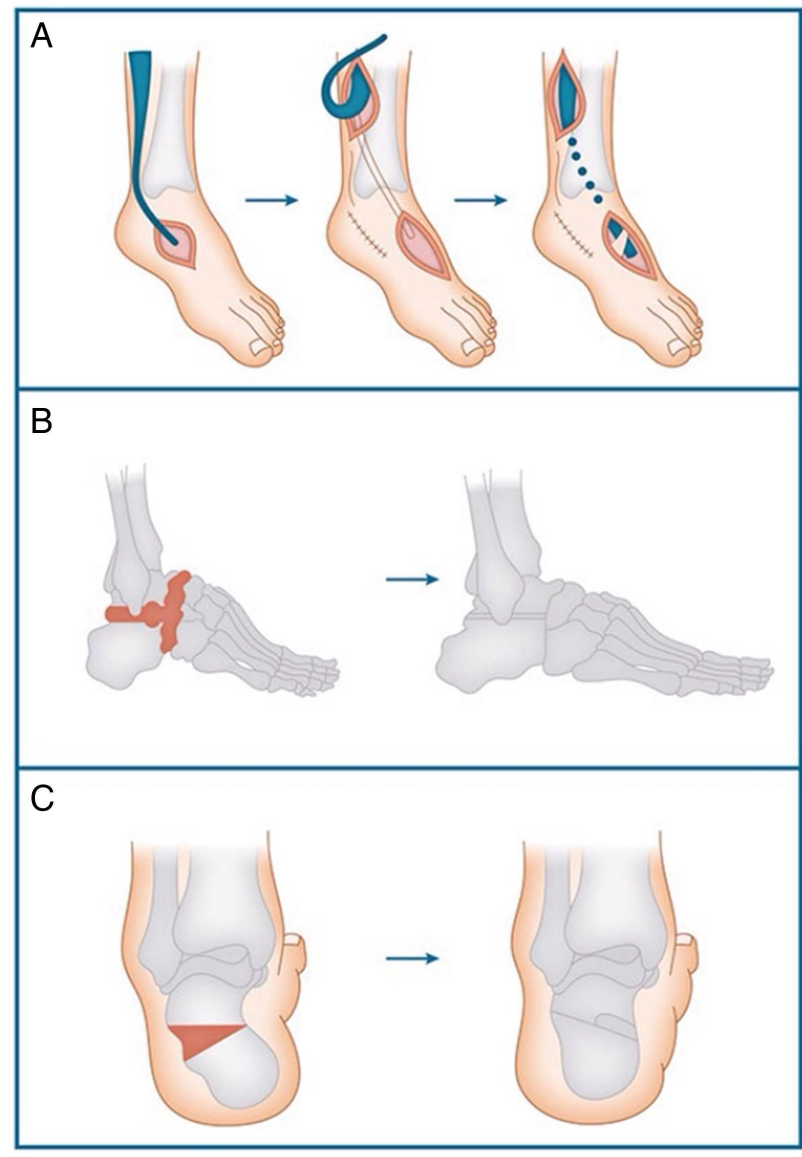

Figure 5 Common foot operations for Charcot-Marie-Tooth disease and related disorders. (A) Various stages of a tendon transfer operation for pes cavus (soft tissue correction) in which the posterior tibialis tendon is transferred to the peroneus brevis tendon, thereby strengthening both the dorsiflexion and eversion forces on the foot and correcting the foot drop and hind foot varus deformity. Other soft tissue correction procedures include Achilles tendon lengthening. (B) Procedure for a triple arthrodesis in which the subtalar, calcaneocuboid and talonavicular joints are fused permitting some movement at the ankle. (C) Procedure of a calcaneal osteotomy required to correct a fixed hind foot varus deformity.

family history and slowly progressive disease course are the strongest clues to a genetic aetiology.

Genetic testing in CMT has been transformed by the introduction of next-generation sequencing. CMT1A due to the $17 \mathrm{p}$ duplication remains the commonest type of CMT and should be tested first in any patient with sporadic or autosomal dominant CMT1 before proceeding to panel or whole exome testing.

The new challenge in CMT and related disorders is in determining the true pathogenic mutation among a handful of novel variants identified in several known disease genes. This can be time consuming and requires a detailed clinical assessment to define the phenotype and where possible the evaluation of affected and unaffected family members.

Although there are no treatments for CMT and related disorders, there is much that can be done to improve a patient's quality of life. Physiotherapists, orthotists and podiatrists can offer advice on orthotics and stretching exercises to prevent Achilles tendon contractures; where there is progressive foot deformity or pain, an orthopaedic foot surgeon with experience in CMT may be consulted.

\section{ONLINE RESOURCES}

Sibyl: http://bioinformatics.ua.pt/sibyl

PON-P2: http://structure.bmc.lu.se/PON-P2/

Predict SNP: http://loschmidt.chemi.muni.cz/predictsnp Meta-SNP: http://snps.biofold.org/meta-snp/

Acknowledgements MMR is grateful to the Medical Research Council (MRC), MRC Centre grant (G0601943), and the National Institutes of Neurological Diseases and Stroke and office of Rare Diseases (U54NS065712) for their support. We are grateful to Shayan Nosrat Jogan for designing and producing figure 5 .

Contributors MMR had the original idea for the manuscript and edited the initial draft. AMR wrote the first draft and MRBE designed the figures and edited the first draft.

Funding Medical Research Council; National Institute of Neurological Disorders and Stroke. AMR has been in receipt of fellowship funding from the National Institutes of Neurological Diseases and Stroke and office of Rare Diseases (U54NS065712) and an IPSEN clinical research fellowship. The INC (U54NS065712) is a part of the NCATS Rare Diseases Clinical Research Network (RDCRN). RDCRN is an initiative of the Office of Rare Diseases Research (ORDR), NCATS, funded through a collaboration between NCATS and the NINDS. This research was also supported by the National Institute for Health Research University College London Hospitals Biomedical Research Centre.

Competing interests None declared.

Provenance and peer review Commissioned; externally peer reviewed. This paper was reviewed by Gareth Llewelyn, Cardiff, UK.

\section{REFERENCES}

1 Reilly MM, Murphy SM, Laura M. Charcot-Marie-Tooth disease. J Peripher Nerv Syst 2011;16:1-14.

2 Houlden H, Laura M, Wavrant-De Vrieze F, et al. Mutations in the HSP27 (HSPB1) gene cause dominant, recessive, and sporadic distal HMN/CMT type 2. Neurology 2008;71:1660-8.

3 Birouk N, Gouider R, Le Guern E, et al. Charcot-Marie-Tooth disease type $1 \mathrm{~A}$ with $17 \mathrm{p} 11.2$ duplication. Clinical and electrophysiological phenotype study and factors influencing disease severity in 119 cases. Brain 1997;120(Pt 5):813-23.

4 Cottenie E, Menezes MP, Rossor AM, et al. Rapidly progressive asymmetrical weakness in Charcot-Marie-Tooth disease type 4J resembles chronic inflammatory demyelinating polyneuropathy. Neuromuscul Disord 2013;23:399-403.

5 Houlden H, King R, Blake J, et al. Clinical, pathological and genetic characterization of hereditary sensory and autonomic neuropathy type 1 (HSAN I). Brain 2006;129(Pt 2):411-25.

6 Michell AW, Laura M, Blake J, et al. GJB1 gene mutations in suspected inflammatory demyelinating neuropathies not responding to treatment. J Neurol Neurosurg Psychiatry 2009;80:699-700

7 Houlden H, Laura M, Ginsberg L, et al. The phenotype of Charcot-Marie-Tooth disease type 4C due to SH3TC2 mutations and possible predisposition to an inflammatory neuropathy. Neuromuscul Disord 2009;19:264-9.

8 Murphy SM, Laura M, Blake J, et al. Conduction block and tonic pupils in Charcot-Marie-Tooth disease caused by a myelin 
protein zero p.Ile112Thr mutation. Neuromuscul Disord 2011;21:223-6.

9 Pelayo-Negro AL, Carr AS, Laura M, et al. An observational study of asymmetry in CMT1A. J Neurol Neurosurg Psychiatry 2014.

10 Liao JP, Waclawik AJ. Nerve root hypertrophy in CMT type 1A. Neurology 2004;62:783.

11 Ishigami N, Kondo M, Nakagawa M. [Case of CharcotMarie-Tooth disease type 1A with increased cerebrospinal fluid proteins and nerve root hypertrophy]. Rinsho Shinkeigaku 2008;48:419-21.

12 Nicholson G, Lenk GM, Reddel SW, et al. Distinctive genetic and clinical features of CMT4J: a severe neuropathy caused by mutations in the PI(3,5)P phosphatase FIG4. Brain 2011;134 (Pt 7):1959-71.

13 Udd B. Molecular biology of distal muscular dystrophiessarcomeric proteins on top. Biochim Biophys Acta 2007; 1772:145-58.

14 Horowitz SH, Schmalbruch H. Autosomal dominant distal myopathy with desmin storage: a clinicopathologic and electrophysiologic study of a large kinship. Muscle Nerve 1994;17:151-60.

15 Pitceathly RD, Murphy SM, Cottenie E, et al. Genetic dysfunction of MT-ATP6 causes axonal Charcot-Marie-Tooth disease. Neurology 2012;79:1145-54.

16 Baets J, Deconinck T, De Vriendt E, et al. Genetic spectrum of hereditary neuropathies with onset in the first year of life. Brain 2011;134(Pt 9):2664-76.

17 Murphy SM, Laura M, Fawcett K, et al. Charcot-Marie-Tooth disease: frequency of genetic subtypes and guidelines for genetic testing. J Neurol Neurosurg Psychiatry 2012;83:706-10.

18 Saporta AS, Sottile SL, Miller LJ, et al. Charcot-Marie-Tooth disease subtypes and genetic testing strategies. Ann Neurol 2011;69:22-33.

19 Thomas PK, Marques W Jr, Davis MB, et al. The phenotypic manifestations of chromosome $17 \mathrm{p} 11.2$ duplication. Brain 1997;120(Pt 3):465-78.

20 Shy ME, Chen L, Swan ER, et al. Neuropathy progression in Charcot-Marie-Tooth disease type 1A. Neurology 2008;70: 378-83.

21 Raeymaekers P, Timmerman V, Nelis E, et al. Duplication in chromosome 17p11.2 in Charcot-Marie-Tooth neuropathy type 1a (CMT 1a). The HMSN Collaborative Research Group. Neuromuscul Disord 1991;1:93-7.
22 Russo M, Laura M, Polke JM, et al. Variable phenotypes are associated with PMP22 missense mutations. Neuromuscul Disord 2011;21:106-14.

23 Chance PF, Alderson MK, Leppig KA, et al. DNA deletion associated with hereditary neuropathy with liability to pressure palsies. Cell 1993;72:143-51.

24 Chance PF. Inherited focal, episodic neuropathies: hereditary neuropathy with liability to pressure palsies and hereditary neuralgic amyotrophy. Neuromolecular Med 2006;8:159-74.

25 Earle N, Zochodne DW. Is carpal tunnel decompression warranted for HNPP? J Peripher Nerv Syst 2013;18:331-5.

26 Ikegami T, Ikeda H, Mitsui T, et al. Novel mutation of the myelin Po gene in a pedigree with Charcot-Marie-Tooth disease type 1B. Am J Med Genet 1997;71:246-8.

27 Verhoeven K, Claeys KG, Zuchner S, et al. MFN2 mutation distribution and genotype/phenotype correlation in Charcot-Marie-Tooth type 2. Brain 2006;129(Pt 8): 2093-102.

28 Zuchner S, Mersiyanova IV, Muglia M, et al. Mutations in the mitochondrial GTPase mitofusin 2 cause Charcot-Marie-Tooth neuropathy type 2A. Nat Genet 2004;36:449-51.

29 Kleopa KA, Scherer SS. Molecular genetics of X-linked Charcot-Marie-Tooth disease. Neuromolecular Med 2006;8:107-22.

30 Dubourg O, Azzedine H, Verny C, et al. Autosomal-recessive forms of demyelinating Charcot-Marie-Tooth disease. Neuromolecular Med 2006;8:75-86.

31 Rossor AM, Polke JM, Houlden H, et al. Clinical implications of genetic advances in Charcot-Marie-Tooth disease. Nat Rev Neurol 2013;9:562-71.

32 Carr AS, Polke JM, Wilson J, et al. MFN2 deletion founder mutation in the UK population. In press.

33 Wright CF, Middleton A, Burton H, et al. Policy challenges of clinical genome sequencing. BMJ 2013;347:f6845.

34 Yagerman SE, Cross MB, Green DW, et al. Pediatric orthopedic conditions in Charcot-Marie-Tooth disease: a literature review. Curr Opin Pediatr 2012;24:50-6.

35 Walker JL, Nelson KR, Heavilon JA, et al. Hip abnormalities in children with Charcot-Marie-Tooth disease. J Pediatr Orthop 1994;14:54-9.

36 Burns J, Crosbie J, Ouvrier R, et al. Effective orthotic therapy for the painful cavus foot: a randomized controlled trial. J Am Podiatr Med Assoc 2006;96:205-11. 